\title{
Agent Based Load Balancing Middleware for Service-Oriented Applications
}

\author{
Jun Wang, Yi Ren, Di Zheng, and Quan-Yuan Wu \\ School of Computer Science, \\ National University of Defence Technology, \\ Changsha, Hunan, China 410073 \\ junwang@nudt.edu.cn
}

\begin{abstract}
Kinds of load balancing middleware have already been applied successfully in distributed computing. However, they don't take the services types into consideration and for different services requested by clients the workload would be different out of sight. Furthermore, traditional load balancing middleware uses the fixed and static replica management and uses the load migration to relieve overload. However, to the complex service-oriented applications, the hosts may be heterogeneous and decentralized at all and load migration is not efficient for the existence of the delay. Therefore, we put forward an Agent based autonomic load balancing middleware to support fast response, hot-spot control and balanced resource allocation among different services. Corresponding simulation tests are implemented and their result $\mathrm{s}$ indicated that this model and its supplementary mechanisms are suitable to complex service-oriented applications.
\end{abstract}

Keywords: Web Service, Service-Oriented Applications, Load Balancing, Adaptive Resource Allocation, Middleware.

\section{Introduction}

In recent years, with the rapid development of e-business, web based applications are developed from localization to globalization, from $\mathrm{B} 2 \mathrm{C}$ to $\mathrm{B} 2 \mathrm{~B}$, from centralized fashion to decentralized fashion and many applications are constructed based on services. The services are executed by using the heterogeneous back-end resources such as high performance systems, mass storage systems, database system etc. However, the applications may be integrated across the Internet by using the services and the distributed services and resources must be scheduled automatically, transparently and efficiently. To service the increasing online clients those transmit a large, often busty, number of requests and provide dependable services with high quality constantly, we must make the distributed computing systems more scalable and dependable. And even under high load, the systems must still support the services as usual. Therefore, we must balance the load of the diverse resources to improve the utilization of the resources and the throughput of the systems. Currently, load balancing mechanisms can be provided in any or all of the following layers in a distributed system: 
- Network-based load balancing: This type of load balancing is provided by IP routers and domain name servers (DNS). However, load balancing at these layers is somewhat limited by the fact that they do not take into account the content of the client requests.

- OS-based load balancing: At the lowest level for the hierarchy, OS-based load balancing is done by distributed operating system in the form of lowest system level scheduling among processors [3, 4].

- Middleware-based load balancing: This type of load balancing is performed in middleware, often on a per-session or a per-request basis. The key enterprise applications of the moment such as astronavigation, telecommunication, and finance all make use of the middleware based distributed software systems to handle complex distributed applications.

There are different realizations of load balancing middleware. For example, stateless distributed applications usually balance the workload with the help of naming service [5]. But this scheme of load balancing just support static non-adaptive load balancing and can't meet the need of complex distributed applications. For more complex applications, the adaptive load balancing schema $[6,7,8]$ is needed to take into account the load condition dynamically and avoid override in some node. However, traditional load balancing middleware uses the fixed and static replica management and load monitoring schemes to relieve overload. But to the complex service-oriented applications, the hosts may be heterogeneous and decentralized at all and load migration is not efficient for the existence of the delay. Therefore, we put forward an Agent based autonomic load balancing middleware to support fast response, hot-spot control and balanced resource allocation among different services.

\section{Architecture of the Load Balancing Middleware}

Our middleware will directly address to the problems by providing load balancing for the service-oriented applications, preventing bottlenecks at the application tier, balancing the workload among the different services and enabling replication of service components in a scalable way to provide more access to the high performance back end resources. The service components are object-based components and they can be distributed or remotely located in different resources. Our load balancing service is a system-level service and it is introduced to the application tier by using IDL $[1,2]$ interfaces. Figure 1 features the core components in our load balancing service as follows:

Service Replica Repository: Instances of services need to register with the Service Group. All the references of the groups are stored in the Service Replica Repository. A service group may include several replicas and we can add or remove replicas to the groups. The main purpose of the service group is to provide a view containing simple information about the references to the locations of all replicas registered with group. The uses need not to know where the replica is located.

Decision Agent Group: The service decision agent acts as a proxy between the client and the dynamic service. It enables transparency between them without letting the client knowing about the multiple distributed service replicas. The agent is in charge 
of getting the best replica for the service and the client requests proceed with their normal procedure such as calling methods from the service replica. The decision agent makes decisions based on the algorithms configured in our load balancing policy according to the Component Configurator design pattern.

Load Monitor: Load monitor collects load information from every load agent within certain time interval. The load information should be refreshed at a suitable interval so that the information provided is not expired. The hosts may have different workload when processing some certain client requests and the workload of the different hosts may fluctuate in different ways. Therefore we suggest an approach that the refresh rate can be adjusted according to the workload of the hosts.

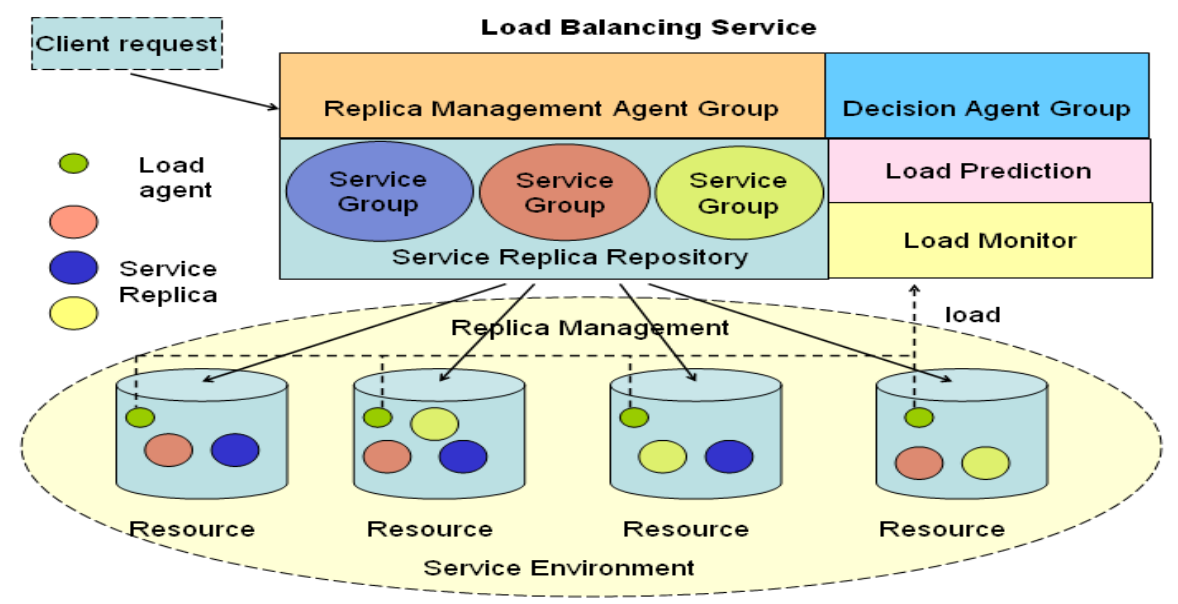

Fig. 1. Components of the Load Balancing Middleware

Load Agent: The purpose of load agent is to provide load information of the hosts it resides when requested by load monitor. As different services might have replicas in the same host, it is hard to presume the percentage of resource is being used by which service at particular moment. Therefore, a general metric is needed to indicate the level of available resources at the machine during particular moment.

Load Prediction: This module use the machine-learning based load prediction method where the system minimizes unpredictable behavior by reacting slowly to changes and waiting for definite trends to minimize over-control decisions.

Replica Management Agent Group: The purpose of these agents is to dynamically manage the replicas to achieve a balance load distribution among different services. In order to carry out service replication, we determine parameters such as initial number of replica, maximum number of replica, when to replicate, where to replicate etc. As different service contains different characteristics such as process time, priority and popularity, we coordinate services in order to avoid monopolizing of resource by popular service and unfair treatment for high priority service.

Several complex tests have been completed, and we can see that by using the Load Agent the overhead of monitoring will be decreased effectively. Furthermore, with the addition of the number of the hosts and the services, the resources can still be 
allocated efficiently and the workload of the resources can be balanced with the help of the Replica Management Agent Group. Especially to the hot-spot services sharing the workloads with the extra replicas is much better than load migration among the overloaded servers.

\section{Conclusions}

Kinds of load balancing middleware have already been applied successfully in distributed computing. However, they don't take the services types into consideration and for different services requested by clients the workload would be different out of sight. Furthermore, traditional load balancing middleware uses the fixed and static replica management and uses the load migration to relieve overload. However, to the complex service-oriented applications, the hosts may be heterogeneous and decentralized at all and load migration is not efficient for the existence of the delay. Therefore, we put forward an Agent based autonomic load balancing middleware to support fast response, hot-spot control and balanced resource allocation among different services. Corresponding simulation tests are implemented and their result s indicated that this model and its supplementary mechanisms are suitable to complex service-oriented applications.

\section{Acknowledgements}

This work was funded by the National Grand Fundamental Research 973 Program of China under Grant No.2005cb321804, the National High-Tech Research and Development Plan of China under Grant No.2004AA112020 and the National Natural Science Foundation of China under Grant No.60603063.

\section{References}

1. Object Management Group, The Common Object Request Broker: Architecture and Specification, 3.0 ed., June 2002.

2. Henning, M., Vinoski, S.: Advanced CORBA Programming With C++. Addison-Wesley Longman, Massachusetts (1999)

3. Chow, R., Johnson, T.: "Distributed Operating Systems and Algorithms", Addison-Wesley Publishing Company (1997)

4. Rajkumar, B.: High Performance Cluster Computing Architecture and Systems, ISBN7.5053-6770-6.2001

5. IONA Technologies, "Orbix 2000." www.iona-iportal.com/suite/orbix2000.htm.

6. Othman, O'Ryan, C., Schmidt, D. C.: The Design of an Adaptive CORBA Load Balancing Service. IEEE Distributed Systems Online(2001)

7. Othman, O., Schmidt, D. C.: Issues in the design of adaptive middleware load balancing. In: ACM SIGPLAN, ed. Proceedings of the ACM SIGPLAN workshop on Languages, Compilers and Tools for Embedded Systems. New York: ACM Press(2001)205-213

8. Othman, O., O'Ryan, C., Schmidt, D.C.: Strategies for CORBA middleware-based load balancing. IEEE Distributed Systems Online(2001) http://www.computer.org/dsonline 